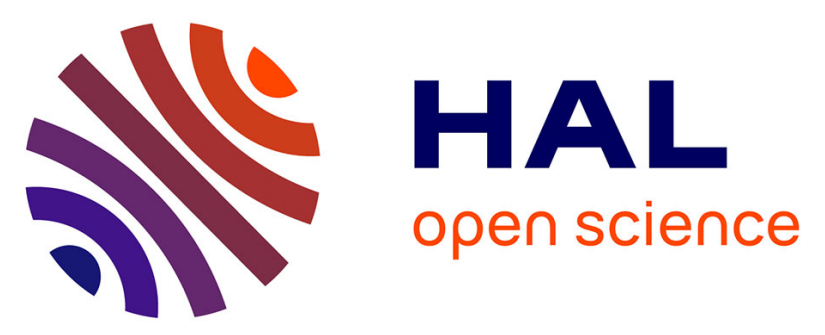

\title{
Improving the Realism in Motion-Based Driving Simulators by Adapting Tilt-Translation Technique to Human Perception
}

Anca Stratulat, Vincent Roussarie, Jean-Louis Vercher, Christophe Bourdin

\section{- To cite this version:}

Anca Stratulat, Vincent Roussarie, Jean-Louis Vercher, Christophe Bourdin. Improving the Realism in Motion-Based Driving Simulators by Adapting Tilt-Translation Technique to Human Perception. 2011 IEEE VIRTUAL REALITY CONFERENCE (VR), 2011, 345 E 47TH ST, NEW YORK, NY 10017 USA, Unknown Region. pp.47-50. hal-01436024

\section{HAL Id: hal-01436024 \\ https://hal.science/hal-01436024}

Submitted on 2 May 2018

HAL is a multi-disciplinary open access archive for the deposit and dissemination of scientific research documents, whether they are published or not. The documents may come from teaching and research institutions in France or abroad, or from public or private research centers.
L'archive ouverte pluridisciplinaire HAL, est destinée au dépôt et à la diffusion de documents scientifiques de niveau recherche, publiés ou non, émanant des établissements d'enseignement et de recherche français ou étrangers, des laboratoires publics ou privés. 


\title{
Improving the Realism in Motion-Based Driving Simulators by Adapting Tilt-Translation Technique to Human Perception
}

\author{
Anca Stratulat ${ }^{* 1,2}$, Vincent Roussarie ${ }^{\dagger 1}$, Jean-Louis Vercher ${ }^{\ddagger 2}$, and Christophe Bourdin ${ }^{\S 2}$ \\ ${ }^{1}$ PSA Peugeot-Citroën, Velizy, France \\ ${ }^{2}$ Institut des Sciences du Mouvement \& CNRS \& Aix-Marseille University, France
}

\begin{abstract}
While modern dynamic driving simulators equipped with six degrees-of-freedom (6-DOF) hexapods and X-Y platforms have improved realism, mechanical limitations prevent them from offering a fully realistic driving experience. Solutions are often sought in the "washout" algorithm, with linear accelerations simulated by an empirically chosen combination of translation and tilt-coordination, based on the incapacity of otolith organs to distinguish between inclination of the head and linear acceleration. In this study, we investigated the most effective combination of tilt and translation to provide a realistic perception of movement. We tested 3 different braking intensities (deceleration), each with 5 inverse proportional tilt/translation ratios. Subjects evaluated braking intensity using an indirect method corresponding to a 2-Alternative-Forced-Choice Paradigm. We find that perceived intensity of braking depends on the tilt/translation ratio used: for small and average decelerations $\left(0.6\right.$ and $\left.1.0 \mathrm{~m} / \mathrm{s}^{2}\right)$, increased tilt yielded an increased overestimation of braking, inverse proportionally with intensity; for high decelerations $\left(1.4 \mathrm{~m} / \mathrm{s}^{2}\right)$, on half the conditions braking was overestimated with more tilt than translation and underestimated with more translation than tilt. We define a mathematical function describing the relationship between tilt, translation and the desired level of deceleration, intended as a supplement to motion cueing algorithms, that should improve the realism of driving simulations.
\end{abstract}

Keywords: Dynamic driving simulator, braking perception, washout algorithm, tilt-coordination, vestibular system, multisensory integration

Index Terms: J.4 [Computer Applications]: SOCIAL AND BEHAVIORAL SCIENCES-Psychology

\section{INTRODUCTION}

Driving simulators have developed rapidly over the last decade, one of the major improvements being the addition of motion to visual, but static, simulations. These dynamic driving simulators attempt to take all aspects of a driving situation into account: the environmental information (visual scenario, auditory cues), the car (physical motion) and the driver (perception of self-motion within the environment). The motion can be on small scale (e.g. 6-DOF hexapods which allow small translations and rotations around the 3 axes) or on large scale (e.g. X-Y platforms which allow long translations up to 40 meters on each axis). Using these motion platforms, the simulations can be based not only on visual and auditory cues, but also

\footnotetext{
*e-mail: anca.stratulat@gmail.com

†e-mail: vincent.roussarie@mpsa.com

¥e-mail: jean-louis.vercher@univmed.fr

$\S$ e-mail: christophe.bourdin@univmed.fr
}

IEEE Virtual Reality 2011

19 - 23 March, Singapore

978-1-4577-0038-5/11/\$26.00 @2011 IEEE on vestibular and proprioceptive cues, which undoubtedly improves realism. But mechanical limitations do not allow for a driving experience comparable with a real situation, especially where there are sustained accelerations.

To overcome these limitations, technical solutions have been sought in other fields, like flight simulation. In the latter, a common solution is the "washout" algorithm [12], now adopted by most motion-based simulators (hexapods). The washout scheme is used to keep the platform within its physical limits. Washout means bringing the simulator cab to its neutral position following the generation of a linear acceleration. The high frequency component of the linear motion cue is produced by translations, while the low frequency component is produced by tilting the motion platform (tilt-coordination). Tilt-coordination is intended to tilt the driver, with a rotation below the vestibular semi-circular canal threshold (to prevent the driver from detecting the rotation), so that the direction of gravity relative to his head is oriented in the same way as the gravito-inertial acceleration (GIA) is oriented during real acceleration (see figure 1). This motion is synchronized with a visual simulation of linear acceleration [9].
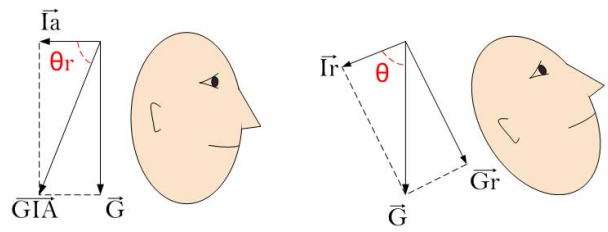

Figure 1: Perceptually equivalent situations, as described in Holly and McCollum [10], are the basis of tilt-coordination technique. The inertial force due to linear acceleration $\left(I_{a}\right)$ together with gravity gives the resulting vector GIA (gravito-inertial acceleration) (a). The vestibular system cannot distinguish between this situation and a tilt of the head, below angular velocity threshold, so that the gravity corresponds to GIA (b). Therefore, a linear acceleration can be simulated by either linear translation or tilt (or a combination of both).

The purpose of the washout algorithm is to offer the driver the best motion cue possible within the physical limits of the simulator. The classical washout algorithm, presented in figure 2 , is characterized by the empirically determined combination of linear high- and low-pass filters [13].

In order to determine the best combination of tilt and translation for such algorithms, it is necessary to take into account human perception of self-motion. Recent studies show that simulator motion affects this perception. For example, in the study of Groen et al. [9] on the use of tilt for the simulation of linear acceleration (tilt-coordination), the perception of linear acceleration depends on angular velocity and tilt angle. But the vestibular system's thresholds for tilt detection(6 deg [6]) and angular velocity (3.7 deg/s [4], between 2 and $4 \mathrm{deg} / \mathrm{s}$ in the study of Groen et al.), impose an upper limit for linear acceleration that can be simulated by tilt without being detected as inclination. Moreover, Groen et al. show 


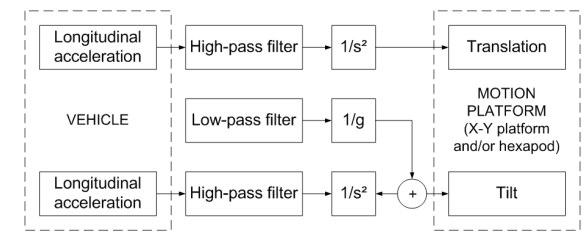

Figure 2: Washout algorithm for a dynamic driving simulator equipped with both hexapod and X-Y motion platform

that there is also a lower limit for motion detection during rotation, which varies inverse-proportionally with motion frequency and proportionally with visual displacement. These results indicate that it is vital to take into consideration not only technical parameters (frequency or level of acceleration), but also the human perception of motion, when simulating believable linear accelerations through tilt-coordination.

The study of Berger el al. [5] on the perception of linear acceleration used not only tilt but also linear translation to simulation motion. They used a 6-DOF hexapod, which allowed both tilt and small horizontal translations and found that a strong visual acceleration presented with consistent platform pitch might be sufficient to produce a good perception of forward acceleration, even if pitch acceleration was above the threshold. Furthermore, the authors suggested that other parameters such as linear motion had mild influence on perception.

Thus, while a simulator is mechanically capable of reproducing a given linear acceleration (positive) or deceleration (negative) by either tilt or translation depending on its frequency (washout algorithm), this does not guarantee a realistic perception of motion in all situations. Further investigation of the process of motion perception is needed, in order to improve the realism of these complex virtual reality devices. The studies presented above raise three questions regarding the use of both tilt and translation for the simulation of linear accelerations and braking (decelerations). First, can the perception of motion be improved by the use of larger translations (up to $10 \mathrm{~m}$ ) in combination with tilt? Second, if so, what the best tilt/translation ratio to simulate a realistic acceleration or deceleration? And third, does this ratio vary with the level of acceleration or deceleration?

Seeking to answer these questions, we conducted an experiment on a dynamic driving simulator equipped with both 6-DOF hexapod and X-Y motion platform, to investigate whether and how the motion perception of different levels acceleration or braking (linear deceleration) is altered when the tilt/translation ratio varies.

\section{General methods}

\subsection{Participants}

There were 24 participants ( 7 women and 17 men), aged from 20 to 60 years (mean 36.87). All gave their informed consent. They all had driving experience (mean 18.14 years), but little driving simulator experience (less than 2 trials). They had normal or correctedto-normal vision.

\subsection{The dynamic driving simulator}

This test was conducted on a dynamic driving simulator equipped with a 6-DOF hexapod placed on a X-Y motion platform. A honeycomb cell placed on the hexapod contained a half-car where the participants sat during the test. The motion envelope given by the hexapod is 0.2 to $0.5 \mathrm{~m}$ on translation and 18 to $23 \mathrm{deg}$ in rotation, while the motion envelope for X-Y platform is 10 and $5 \mathrm{~m}$ (on longitudinal and transversal directions, respectively). The visual scene was displayed on 3 flat screens with a $160 \mathrm{deg}$ x 25 deg (horizontal $\mathrm{x}$ vertical) field of view. Sound was via surround system (6 loudspeakers and a sub-woofer).

\subsection{Protocol and experimental design}

The subjects were passive riders, meaning that they were subjected to a pre-defined scenario. We chose an impoverished visual scenario in order to give more weight to the other sensory cues, especially vestibular cues.

The visual environment consisted of a single-lane straight road surrounded by empty green fields. The road and the fields had no visible texture, to prevent participants form detecting their spatial position through visual landmarks. A white line drawn transversally on the pavement was placed $200 \mathrm{~m}$ from the starting position of the car. A wall was placed transversal to the road at a certain distance from the line. From the starting point up to the white line, the road was bordered by trees to produce optic flow during the displacement. After the white line, there were some trees placed randomly within the green field but at long distance from the road, so that their position was visible only in the peripheral visual field of the driver (thus allowing speed perception but no spatial positioning). After the white line, therefore, the visual information remained constant.

The car drove off towards the wall at constant speed. After 20 $\mathrm{sec}$, the car crossed the line and started to brake, and the wall disappeared instantaneously. The car braked for $4 \mathrm{sec}$ then came to a full stop. Although the car always stopped right before the wall, touching it with its bumper, the participants were not informed of this. Their task was to evaluate the braking intensity, by answering the following question: "Did the car stop before or after the wall?", which corresponds to a two-alternative-forced-choice (2AFC) paradigm.

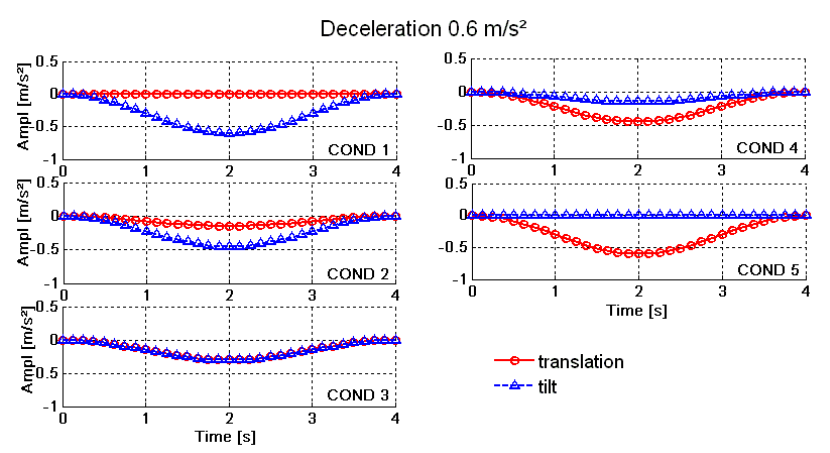

Figure 3: The dynamic stimulation of braking (motion platform) for a level of deceleration was presented as 5 combinations of 2 sinusoidal signals, translation (red line, dots) and tilt (blue line, triangles). The sum of the two signals was equal with the respective level of deceleration. The 5 conditions for $0.6 \mathrm{~m} / \mathrm{s}^{2}$ are presented here.

The dynamic stimulation, reproducing the physical motion of the car, consisted in simulating the braking through tilt and translation. Due to the non-linearity of human perception, three levels of deceleration were tested. Braking time was constant at $4 \mathrm{sec}$, but we varied the level of deceleration. Therefore the initial velocity and the braking distance varied according to the level of deceleration.

Each of the three decelerations was dynamically simulated on 5 combinations of tilt and translation (tilt/translation ratios) (see figure 3) described in table 1. Each combination of deceleration with specific a tilt/translation ratio was repeated 3 times, giving a total of 45 trials per subject, presented randomly in 4 series.

\subsection{Data analysis}

The median of braking evaluation was calculated for each participant and each condition, to enable us to compute the psychometric 
Deceleration $0.6 \mathrm{~m} / \mathrm{s}^{2}$

\begin{tabular}{ccccc}
\hline \multicolumn{5}{c}{ Initial visual speed: $13.88 \mathrm{~m} / \mathrm{s}$ - Braking distance: $16 \mathrm{~m}$} \\
\hline Condition & Translation & \multicolumn{3}{c}{ Tilt } \\
\hline & $\mathrm{m} / \mathrm{s}^{2}$ & $\mathrm{~m} / \mathrm{s}^{2}$ & $\mathrm{deg} / \mathrm{s}$ & $\mathrm{deg}$ \\
\hline 1 & 0.00 & 0.60 & 2.75 & 3.50 \\
2 & 0.15 & 0.45 & 2.06 & 2.63 \\
3 & 0.30 & 0.30 & 1.38 & 1.75 \\
4 & 0.45 & 0.15 & 0.69 & 0.88 \\
5 & 0.60 & 0.00 & 0.00 & 0.00
\end{tabular}

\begin{tabular}{|c|c|c|c|c|}
\hline \multicolumn{5}{|c|}{ Deceleration $1.0 \mathrm{~m} / \mathrm{s}^{2}$} \\
\hline \multicolumn{5}{|c|}{ Initial visual speed: $16.66 \mathrm{~m} / \mathrm{s}$ - Braking distance: $26 \mathrm{~m}$} \\
\hline Condition & Translation & & Tilt & \\
\hline & $m / s^{2}$ & $\mathrm{~m} / \mathrm{s}^{2}$ & $\mathrm{deg} / \mathrm{s}$ & deg \\
\hline 1 & 0.00 & 1.00 & 4.60 & 5.85 \\
\hline 2 & 0.25 & 0.75 & 3.44 & 4.39 \\
\hline 3 & 0.50 & 0.50 & 2.29 & 2.92 \\
\hline 4 & 0.75 & 0.25 & 1.15 & 1.46 \\
\hline 5 & 1.00 & 0.00 & 0.00 & 0.00 \\
\hline
\end{tabular}

\begin{tabular}{|c|c|c|c|c|}
\hline \multicolumn{5}{|c|}{ Deceleration $1.4 \mathrm{~m} / \mathrm{s}^{2}$} \\
\hline \multicolumn{5}{|c|}{ Initial visual speed: $19.44 \mathrm{~m} / \mathrm{s}$ - Braking distance: $36 \mathrm{~m}$} \\
\hline Condition & Translation & & Tilt & \\
\hline & $\mathrm{m} / \mathrm{s}^{2}$ & $m / s^{2}$ & $\mathrm{deg} / \mathrm{s}$ & deg \\
\hline 1 & 0.00 & 1.40 & 6.44 & 8.21 \\
\hline 2 & 0.35 & 1.05 & 4.83 & 6.14 \\
\hline 3 & 0.70 & 0.70 & 3.21 & 4.09 \\
\hline 4 & 1.05 & 0.35 & 1.61 & 2.05 \\
\hline 5 & 1.40 & 0.00 & 0.00 & 0.00 \\
\hline
\end{tabular}

Table 1: Dynamic simulation of braking by a combination of tilt and translation

function for each deceleration, using a Probit model. This method of analysis, adapted for $2 \mathrm{AFC}$ paradigms (binomial response proto$\mathrm{col}$ ), allowed us to determine the point of subjective equality (PSE) [11] for each deceleration, corresponding to a tilt/translation ratio perceived as being the most realistic (i.e. the ratio for which the driver cannot decide whether the car stopped before or after the wall).

\section{Results}

The participants detected differences in braking intensity between trials for the same level of deceleration, but they could not attribute this change to the variation of tilt or translation used to simulate the deceleration. Moreover, even when, in some cases, they detected the pitch tilt of the car, they considered it as a natural motion of the car during braking, as shown by the fact that none of the participants experienced motion sickness. Moreover, none of them realized that the car always stopped just in front of the wall, touching it with its bumper, since there was no visual texture and the wall disappeared as soon as braking began.

The results show an increased overestimation of braking proportional with the increases in the quantity of tilt used to simulate the linear deceleration (see figure 4). For small and average decelerations $\left(0.6\right.$ and $\left.1.0 \mathrm{~m} / \mathrm{s}^{2}\right)$, the increased use of tilt yielded an increased overestimation of the braking, inverse proportionally with the intensity of the braking. For high decelerations $\left(1.4 \mathrm{~m} / \mathrm{s}^{2}\right)$, on half of the conditions there was an overestimation of the braking when we used more tilt than translation and an underestimation when we used more translation than tilt.

In order to determine the most realistic tilt/translation ratio, a psychometric function (Probit) was computed for each level of deceleration: $P S E_{0.6}=8.85, P S E_{1.0}=4.99, P S E_{1.4}=3.34$. The results suggest that the PSE depends on the level of deceleration (see figure 5).

The three curves presented in figure 5 describe the evolution of braking perception in terms of tilt/translation ratios. The ratios used in our experiment are 1, 2, 3, 4 and 5. Our results show that the

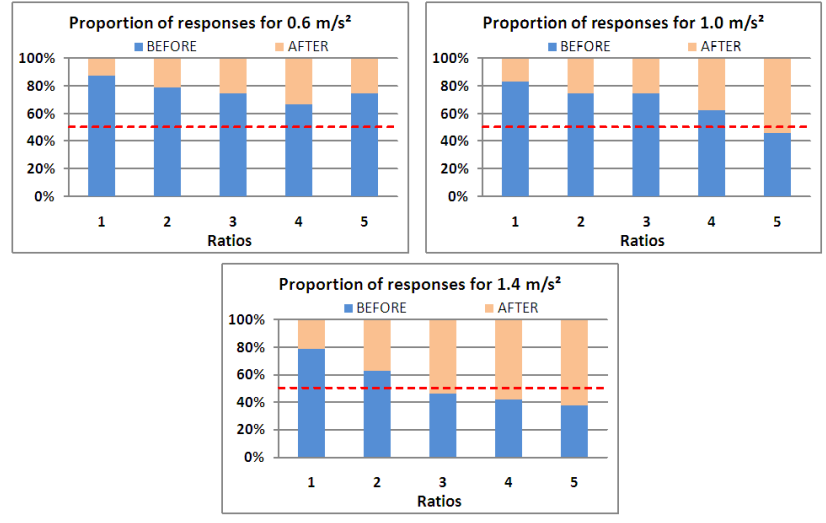

Figure 4: The number participants answering BEFORE or AFTER for each condition. For each participant only one answer was retained (the median value of the 3 trials).

deceleration of $0.6 \mathrm{~m} / \mathrm{s}^{2}$ is systematically overestimated, whatever the tilt/translation ratio used (green curve). For 1.0 (red curve), the braking is well estimated when simulated by pure translation (ratio 5), but overestimated in all other cases. For $1.4 \mathrm{~m} / \mathrm{s}^{2}$ the braking is well estimated for an approximatively $41 / 59 \%$ tilt/translation ratio (where the blue curve crosses the threshold line). When more than $41 \%$ of the deceleration is simulated by the tilt, the braking is overestimated, while when more than $60 \%$ of translation is used, the braking is underestimated.

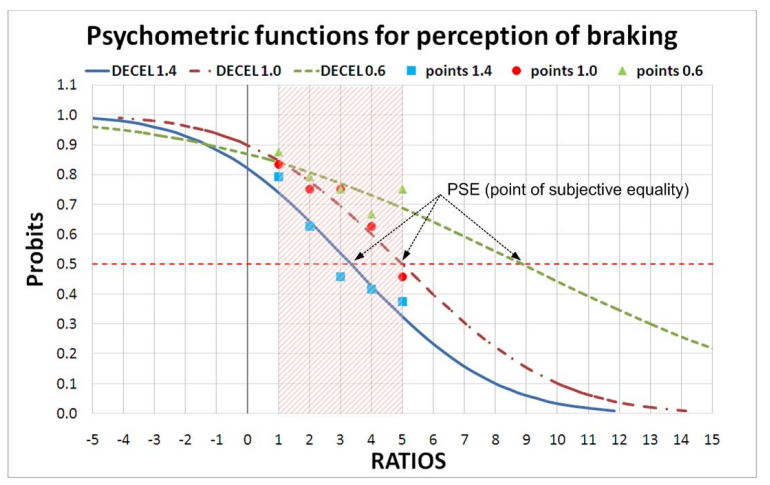

Figure 5: The psychometric functions calculated for each deceleration level. A response probability of 1 means that $100 \%$ of the subjects are sure that the car stopped BEFORE the wall (overestimation) while 0 means AFTER (underestimation). The PSE is the point of the curve with a probability of 0.5 . The dashed zone defines the tested ratios. Note that the lower deceleration level cannot be realistically simulated at the scale factor of 1 used here.

\section{Discussion}

In the present study we examined how the perception of three levels of deceleration on a dynamic driving simulator is influenced by changes in the ratio between tilt and translation. Our results suggest that braking intensity is perceived as directly proportional to the quantity of tilt used to simulate the deceleration, and inversely proportional to the amount of translation. Thus, the most realistic tilt/translation ratio, described in our results by the PSE, depends on the level of simulated deceleration required. An explanation for these differences in the perception of motion on a dynamic driving simulator should probably be sought in the functionality of the vestibular system and the interactions among all the sensory cues involved in the process of multi-sensory integration during braking. 
From the vestibular point of view, the different tilt/translation ratios for a given deceleration are considered to be perceptually equivalent [10], but only if both movements are produced in the dark in a static way (constant stimuli) and below the detection thresholds of the semi-circular canals of the vestibular system (3.7 $\mathrm{deg} / \mathrm{sec}$ for angular velocity [4] and $6 \mathrm{deg}$ for inclination [6]). This principle of equivalence, first explained by Albert Einstein [7], and based on the tilt-translation ambiguity given by the vestibular system in the dark[3], represents the basis of the washout algorithm. But on a driving simulator, most of the movements are neither in the dark nor constant.

Our results suggest that taking this equivalence for granted when choosing the parameters for virtual reality devices is not the best solution. Even if the rotations are kept below the detection thresholds (so here, $0.6 \mathrm{~m} / \mathrm{s}^{2}$ ), it seems that the final perception of motion is influenced not only by vestibular stimuli, but by the integration of multiple sensory signals. Moreover, these thresholds may be modified by the interaction of multiple sensory cues: studies on bimodal perception on audio-visual [1] or visuo-vestibular [2] integration show that threshold for combined conditions are smaller than the thresholds for unimodal perception. Hence, in our case, in some of the conditions, the tilt could have been detected by the participants, even if theoretically the it was below the detection thresholds of the vestibular system. Nevertheless, this excessive tilt does not explain the differences in perception between ratios, given the fact that, for ratio 1 (pure tilt), all decelerations had the same effect, regardless of the different angular velocities or tilt angles. But a plausible explanation for this difference in the perception of different ratios of the same level of deceleration might be the presence of proprioceptive cues (motion of the head, contact of the seatbelt with the body, cervical proprioception, neck proprioception etc.) that may add non-vestibular information.

This interaction between vestibular and proprioceptive cues does not, however, fully explain the second part of our results: that is, why the most realistic tilt/translation ratio varies with deceleration level. Perception of different braking intensities may well be influenced by proprioceptive cues, but we cannot discount the effect of the varying visual and auditory cues from one deceleration to another. It is impossible to completely isolate proprioceptive cues during this kind of simulation, and therefore we can only assume that they contributed, together with vestibular, auditory and visual cues, to braking perception. Further attention to the question of multisensory integration would help elucidate these points.

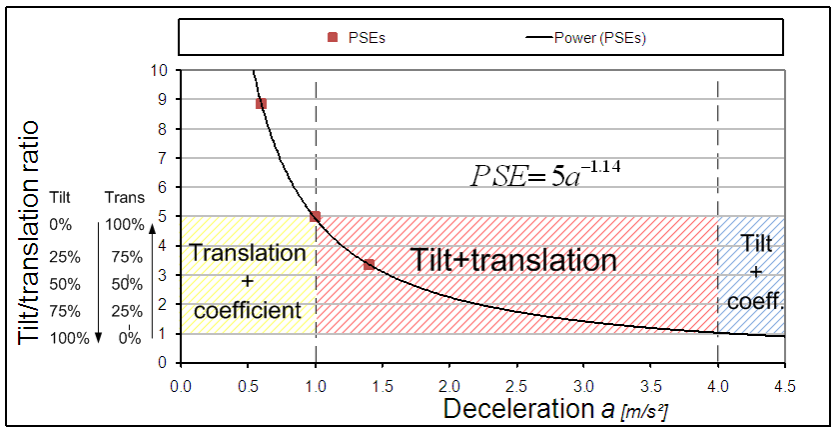

Figure 6: The relationship between tilt/translation ratios and simulated accelerations.

Nevertheless, our results suggest that the classical washout algorithm needs to be adapted not only to the mechanical limitations of the virtual reality device, but also to the non-linearity of the human perceptual system, e.g. different ratios should be used depending on the intended level of deceleration. Consequently, we propose an empirically found data-driven fitting model to determine the tilt/translation ratio according to the level of deceleration in or- der to improve the realistic perception of braking on a complex virtual device like a dynamic driving simulator. Fig. 6 describes the relationship between tilt, translation and the level of simulated deceleration required ( $a$ is the desired deceleration to simulate). For $1 \leq a \leq 4$, the quantity of tilt and translation to be used for the simulation of $a$ is derived from PSE function. For $a<1$, only translation may be used, but with a coefficient (see [8]). The same, for $a>4$, only tilt may be used, but also with a coefficient.

Further studies are needed to propose a functional model operating over a wider range of motion (linear and lateral accelerations). Such a model would be a valuable improvement of the motion cueing algorithms for driving simulators.

\section{Conclusion}

If driving simulators are to be made even more realistic, the human perceptual process needs to be better taken into account. Our results here suggest that the perceived intensity of braking depends on the tilt/translation ratio used to simulate the level of deceleration. The mathematical function of this relationship that we propose is a first step towards determining the most effective tilt/translation ratio for a given deceleration level, offering the promise of improved realism in tomorrows driving simulators.

\section{ACKNOWLEDGEMENTS}

The authors would like to thank Marjorie Sweetko for the language editing of this paper. This work was supported in part by a $\mathrm{PhD}$ grant from ANRT (Association Nationale de la Recherche et de la Technologie) and PSA Peugeot-Citroën.

\section{REFERENCES}

[1] D. Alais and D. Burr. The ventriloquist effect results from nearoptimal bimodal integration. Current Biology, 14(3):257-262, Feb 2004.

[2] D. E. Angelaki, Y. Gu, and G. C. DeAngelis. Multisensory integration: psychophysics, neurophysiology, and computation. Current Opinion in Neurobiology, 19:452-458, 2009.

[3] D. E. Angelaki, M. Wei, and D. M. Merfeld. Vestibular discrimination of gravity and translational acceleration. Annals of the New York Academy of Sciences, 942:114-127, Oct 2001.

[4] A. Benson. Perception and control of self motion, chapter Sensory functions and limitations of the vestibular systems, pages 145-170. R. Warren and A.H. Wertheim, 1990.

[5] D. Berger, J. Schulte-Pelkum, and H. Bulthoff. Simulating believable forward accelerations on a stewart motion platform. In ACM TRANSACTIONS ON APPLIED PERCEPTION, 2010.

[6] L. Bringoux, V. Nougier, P.-A. Barraud, L. Marin, and C. Raphel. Contribution of somesthetic information to the perception of body orientation in the pitch dimension. The Quarterly Journal of Experimental Psychology, 56(5):909-923, Jul 2003.

[7] A. Einstein. The meaning of relativity. Princeton University Press, Princeton, New Jersey, 1956.

[8] E. Groen, C. M. Valenti, and R. Hosman. Psychophysical thresholds associated with the simulation of linear acceleration. In AIAA, 2000.

[9] E. L. Groen and W. Bles. How to use body tilt for the simulation of linear self motion. Journal of Vestibular Research, 14(5):375-385, 2004.

[10] J. E. Holly and G. McCollum. The shape of self-motion perceptioni. equivalence classification for sustained motions. Neuroscience, 70(2):461-486, Jan 1996.

[11] N. A. Macmillan and C. D. Creelman. Detection theory: A User's guide. Lawrence Elbaum Associates, 2005.

[12] M. A. Nahon and L. D. Reid. Simulator motion-drive algorithm: A designer's perspective. Journal of Guidance, Control, and Dynamics, 13-2:356-362, 1990.

[13] S.-C. Wang and L.-C. Fu. Predictive washout filter design for vr-based motion simulator. In Proceedings of IEEE International Coriference on Systems, Man and Cybernetics, 2004. 